\title{
Profile of Nodal Tuberculosis at a Tertiary Care Centre, Gwalior, India
}

\author{
Authors \\ Dr Jyoti Priyadarshini Shrivastava, Dr Poonam Woike, Dr LokeshTripathi, \\ Dr Rajesh Gaur, Dr K.S Mangal \\ Department Of Pathology, G.R Medical College, Gwalior, M.P, India \\ Corresponding Author \\ Dr Jyoti Priyadarshini Shrivastava \\ Residence-155.Saraswati Nagar, University Road, City Centre, Gwalior (M.P)-474011 \\ Email-drpriyajyoti22@gmail.com,Phone No.-+919425029163
}

\begin{abstract}
Lymphadenitis is the most common extra pulmonary manifestation of tuberculosis. Tuberculous lymphadenitis is considered to be the local manifestation of the systemic disease. A high index of suspicion is needed for the diagnosis of tuberculous lymphadenitis which is known to mimic a number of pathological conditions. Cytomorphology proves to be a valuable tool in diagnosing these cases. The aim of our study is to evaluate the utility and limitations of fine needle aspiration cytology and various cytomorphological presentations in reference to tuberculous lymphadenitis. In a study period from January 2010 - December 2014,1188 superficial lymphnodes, were selected for FNA and subjected to cytological evaluation by Giemsastained smears. Incidence of tuberculous lymphadenitis was 54.95\% ( $p$ value-0.035). Cervical region was the commonest site of involvement with solitary lymphadenopathy as the most common presentation in contrast to matted lymph nodes as reported by others. Fine needle aspiration cytology is a safe, cheap, rapid outpatient procedure requiring minimal instrumentation and is highly sensitive to diagnose tubercular lymphadenitis.

Key Words- Tuberculosis, Cytomorphology, Lymphadenitis, Epithelioid cells
\end{abstract}

\section{Introduction}

Tuberculosis, MTB, or TB (short for tubercle bacillus), in the past also called phthisis, phthisis pulmonalis, orconsumption, is a widespread, infectious disease caused by various strains of mycobacteria, usually Mycobacterium tuberculosis. ${ }^{[1]}$ Tuberculosis (TB) has been a major cause of suffering and death since times immemorial. Thought to be one of the oldest human diseases, the history of TB is almost as old as mankind ${ }^{[2]}$.

TB is worldwide in distribution, but is particularly more prevalent in Asia and Africa. According to a 2008 World Health Organization (WHO) report, 9.2 million cases were detected and 1.7 million people lost their lives due to TB the world over. India, China, Indonesia, South Africa and Nigeria rank first to fifth respectively in terms of absolute 
numbers of cases ${ }^{[3]}$. One-third of the world's population is thought to have been infected with $M$. tuberculosis, ${ }^{[4]}$ and new infections occur in about $1 \%$ of the population each year. ${ }^{[5]}$ In 2007, an estimated 13.7 million chronic cases were active globally, ${ }^{[6]}$ while in 2013 , an estimated 9 million new cases occurred. ${ }^{[7]}$ In 2013 there were between 1.3 and 1.5 million associated deaths, ${ }^{[7][8]}$ most of which occurred in developing countries. ${ }^{[9]}$ The total number of tuberculosis cases has been decreasing since 2006, and new cases have decreased since $2002 .{ }^{[9]}$ More people in the developing world contract tuberculosis because of a poor immune system, largely due to high rates of HIV infection and the corresponding development of AIDS. ${ }^{[10]}$ India has the highest TB burden accounting for one fifth of the global incidence. Tuberculous lymphadenitis (or tuberculous adenitis) is a chronic specific granulomatous inflammation of the lymph node with caseation necrosis. The characteristic morphological element is the tuberculous granuloma (caseating tubercule). This consists of giant multinucleated cells (Langhans cells), surrounded by epithelioid cells aggregates, T cell lymphocytes andfibroblasts. Granulomatous tubercules eventually develop central caseous necrosis and tend to become confluent, replacing the lymphoid tissue. Stages of Tubercular Lymphadenitis-Lymphadenitis, Periadenitis, Coldabscess, 'Collar stud' abscess and Sinus.

Tuberculosis may infect any part of the body, but most commonly occurs in the lungs (known as pulmonary tuberculosis). ${ }^{[11]}$ Extrapulmonary TB occurs when tuberculosis develops outside of the lungs, although extrapulmonary TB may coexist with pulmonary $\mathrm{TB}$, as well. ${ }^{[11]}$

General signs and symptoms include fever, chills, night sweats, loss of appetite, weight loss, and fatigue. ${ }^{[11]}$ Significant nail clubbing may also occur. $^{[12]}$

In $15-20 \%$ of active cases, the infection spreads outside the lungs, causing other kinds of $\mathrm{TB} .{ }^{[13]}$ These are collectively denoted as "extrapulmonary tuberculosis". ${ }^{[14]}$ Extrapulmonary TB occurs more commonly in immunosuppressed persons and young children. In those with HIV, this occurs in more than $50 \%$ of cases. ${ }^{[14]}$ The term "extrapulmonary TB" has been used to describe the isolated occurrence of TB at body sites other than the lung. The most common sites of extrapulmonary tuberculosis consist of lymphatic, genitourinary, bone and joint, and central nervous system involvement, followed by peritoneal and other abdominal organ involvement [15]. Tubercular lymphadentis is a common manifestation of extrapulmonary tuberculosis. Fine needle aspiration cytology (FNAC) being a simple outpatient diagnostic procedure is well accepted by patients and has practically no complications $^{(16)}$. It provides an alternative to excision biopsy for lymph nodes and is an easy procedurefor collection of material for cytomorphological , bacteriological examination and immunochemistry ${ }^{(17)}$.

\section{Materials and methods}

The study was conducted in Cytopathology section of Department of Pathology of our centre. Patients who visited OPD of our Hospital, with complaints of superficial lymphadenopathy were sent for FNAC for definitive diagnosis. FNAC was performed using 23 gauge needle attached to $20 \mathrm{ml}$ syringe. In each procedure, an average of 2 to 3 passes is usually performed and aspirated materials smeared into 2-3 slides. Slides were stained with May Grunwald Giemsa (MGG) stain and using D.P.X mountant were prepared for cytological examination. The reporting was done by experienced cytopathologists and the data of reported cases over past five years (2010-2014) were collected and categorized.

The data from the 2010-2014 were retrieved, compiled, summarized and statistically analyzed by frequency distribution and percentage proportion.

Chi square test was applied to know the significant value ( $\mathrm{p}$ value). 
Limitations of the study- The study could only be carried out in the patients who visited the OPD of our hospital, so the actual profile in this region was not established due to the undiagnosed cases in this region.

\section{Results}

In this 5 years study a total of 1188 patients with superficial lymphadenopathy were examined. In our study, the most common superficial lymphadenopathy is cervical $(90.15 \%)$, followed by axillary $(6.98 \%)$ and lastly inguinal $(2.3 \%)$. Out of total 1188 cases examined 641(53.95\%) were positive for tubercular infection. The spectrum of various lymphadenopathy yearwise from $2010-2014$ and cases positive for tuberculosis are shown in table $1 \& 2$ \& Fig no. 1. Male to female ratiois shown in figure number 2. Lymph node Tuberculosis was reported more in females than males every year and the number is increasing. Further, Table no. 3 shows the distribution of tubercular lymphadenopathy in various age groups.

\section{Discussion}

Lymphadenopathy is a clinical manifestation of regional or systemic disease which serves as an excellent clue to the underlying disease. It can arise either from benign or malignant causes depending upon the geographical condition and socioeconomic set up (Ahmad et al., 2009) ${ }^{[18]}$. In the present study patients presenting with superficial lymphadenopathy, who visited the OPD of J.A Hospital Gwalior were sent for cytopathological examination for final diagnosis. A total number of 1188 cases were examined from January 2010- December 2014 and from the anatomical findings the results show that cervical lymphadenopathy was the most common finding followed by axillary and inguinal lymphadenopathy. India is a developing country and the prevalence of tuberculosis is very high here. Tuberculosis mainly presents as cervical lymphadenopathy without any pulmonary manifestation in most of the cases.
Clinically, in our study, cervical region was the most commonly affected region, involved in $90 \%$ of cases. Cervical nodes were also found to be most frequently involved in other studies ${ }^{[19][20]}$. It can be noted from the above data that the prevalence of tubercular lymphadenopathy in our region is $54.95 \%$ ( $\mathrm{p}$ value- 0.035 ) which is similar to results in other study done by Ruchiet $a l^{[19]}$. Another study done by Chawla et al ${ }^{[21]}$ showed much lower incidence of tubercular lymphadenopathy $31.7 \%$. The present study showed the maximum prevalence of tuberculosis lymphadenitis in the $2^{\text {nd }}$ and $3^{\text {rd }}$ decade. Mostly in the age group between 16-45 years. Similar pattern of age distribution has also been in some studies ${ }^{[19,21]}$. Mohapatra and Janmeja ${ }^{[22]}$ had reported the maximum incidence seen in 2nd decade followed by 3 rd decade. The youngest patient in our series was a 2 years old child while the oldest one was 74 years. The disease appears to be less common in extremes of age group. Narang et al., ${ }^{[23]}$ had reported minimum age of 4 years with maximum being 70 year. The present study, found that the females were more commonly affected than males (male to female ratio was $1: 1.3$. The higher incidence of disease among females may be due to the low immunity of Indian females, particularly those belonging to low socioeconomic strata and those in reproductive age group. Paliwal et al., [20], Khajuria et al., ${ }^{[19]}$, Narang et al., ${ }^{[23]}$,Mohapatra and Janmeja [22] also noted the female preponderance in their studies. In our study, patients most commonly presented with single nodes $(456,71.1 \%)$, while $185(28.8 \%)$ patients presented with multiple cervical nodes. Paliwal et al., ${ }^{[20]}$ had reported $7.2 \%$ patients presented with bilateral cervical nodes and $19.2 \%$ with multiple unilateral nodes.

For cytopathological diagnosis of tubercular lymphadenopathy, the diagnostic features are presence of epithelioid cells, giant cells(Langhans and Foreign body type), caseation necrosis,collar of lymphocytes, fibroblasts with or without granuloma formation(Fig.No.3).Studies by Gomes 
et al ${ }^{[24]}$, Das et al ${ }^{[25]}$ also followed the same cytological parameters for diagnosis of tubercular lymphadenitis.

In this study, based on cytological findings, we categorized the lesions into four categories: a) Cases with only caseous necrosis, b) Cases with caseous necrotic material and degenerated inflammatory cells, c) Cases with caseous necrotic material and epithelioid giant cell granulomas and giant cells and d) cases with reactive lymphoid cells and epithelioid giant cell granulomas. From the above data it can also be noted that the cases positive for tubercular lymphadenopathy increased from 2010 to 2014 which shows increase in prevalence of tuberculosis and it's infectivity in our region. Therefore, FNAC of every suspicious Lymph node is mandatory and it is proved to be a reliable tool for diagnosis of tubercular lymphadenitis and it's early treatment.

\section{Conclusion}

FNAC is a reliable, quick and economical investigating modality in tubercular lymphadenopathy. It provides a high degree of accuracy in diagnosing VARIOUS conditions including tuberculosis. Timely diagnosis is helpful in reducing morbidity and mortality of tuberculosis. In a developing country like India, where there is high prevalence of tuberculosis FNAC serves as a rapid, reliable and economical diagnostic method in order to get a prompt result which is beneficial for the patient and also for reducing the prevalence of tuberculosis in the country.

Table No. 1.Year Wise Distribution Of Lymphadenopathy-

\begin{tabular}{|l|c|c|c|c|}
\hline Year & Cervical & Axillary & Inguinal & Total \\
\hline 2010 & $190(81.54 \%)$ & $35(15.02 \%)$ & $08(3.4 \%)$ & 233 \\
\hline 2011 & $160(68.66 \%)$ & $20(10.8 \%)$ & $05(2.7 \%)$ & 185 \\
\hline 2012 & $230(95 \%)$ & $10(4.1 \%)$ & $02(0.8 \%)$ & 242 \\
\hline 2013 & $243(94 \%)$ & $12(4.65 \%)$ & $03(1.16 \%)$ & 258 \\
\hline 2014 & $248(95.3 \%)$ & $06(2.30 \%)$ & $05(1.9 \%)$ & 260 \\
\hline
\end{tabular}

Table no.2 Cases positive for tuberculosis-

\begin{tabular}{|l|c|}
\hline Year & Cases positive for Tuberculosis \\
\hline 2010 & $120(51.5 \%)$ \\
\hline 2011 & $107(57.8 \%)$ \\
\hline 2012 & $135(55.7 \%)$ \\
\hline 2013 & $138(53.4 \%)$ \\
\hline 2014 & $141(54.2 \%)$ \\
\hline
\end{tabular}

Table no.3 Age group wise distribution of cases positive for tuberculosis-

\begin{tabular}{|c|c|c|c|}
\hline Year & $0-15$ years & $16-45$ years & $>45$ years \\
\hline 2010 & 20 & 73 & 27 \\
\hline 2011 & 12 & 80 & 15 \\
\hline 2012 & 22 & 87 & 26 \\
\hline 2013 & 16 & 95 & 27 \\
\hline 2014 & 19 & 100 & 22 \\
\hline
\end{tabular}




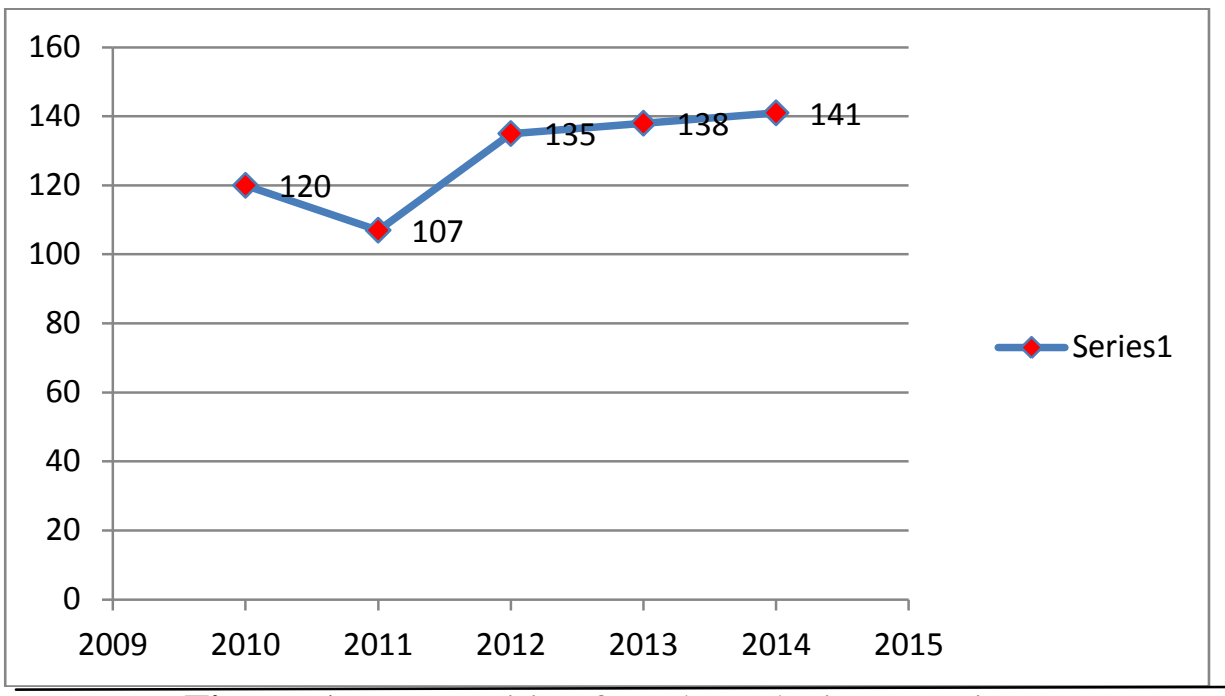

Fig. no.1 cases positive for tuberculosis year wise.

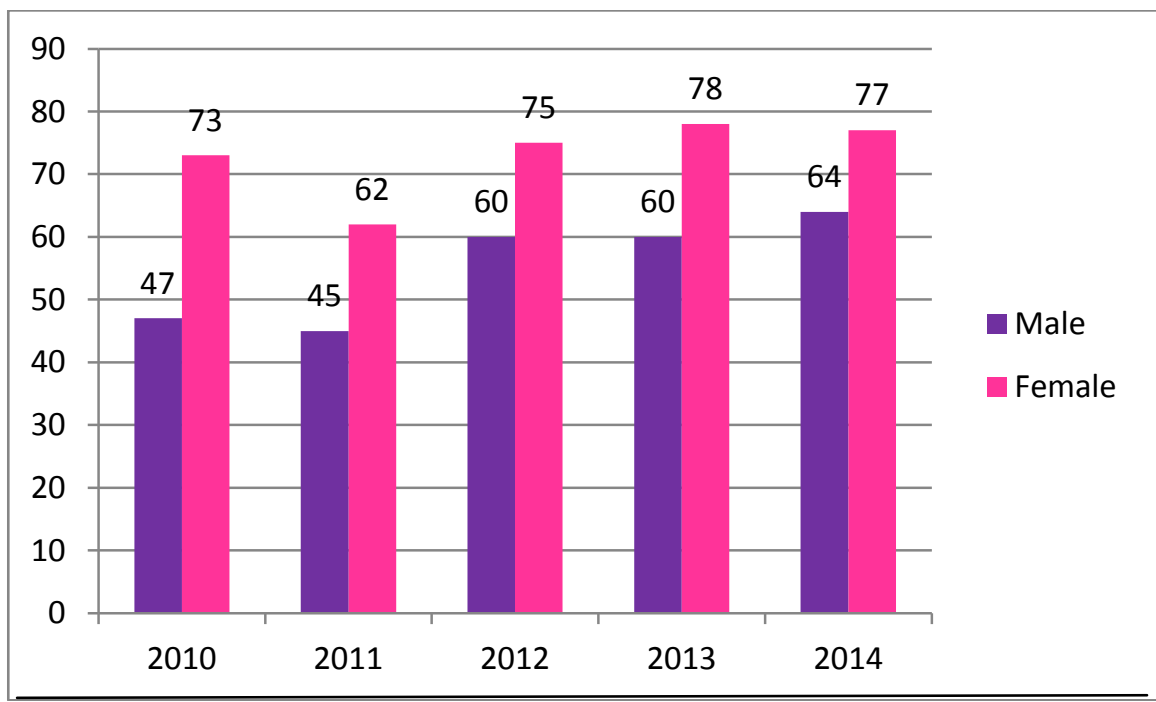

Fig. no.2 Male Female Distribution

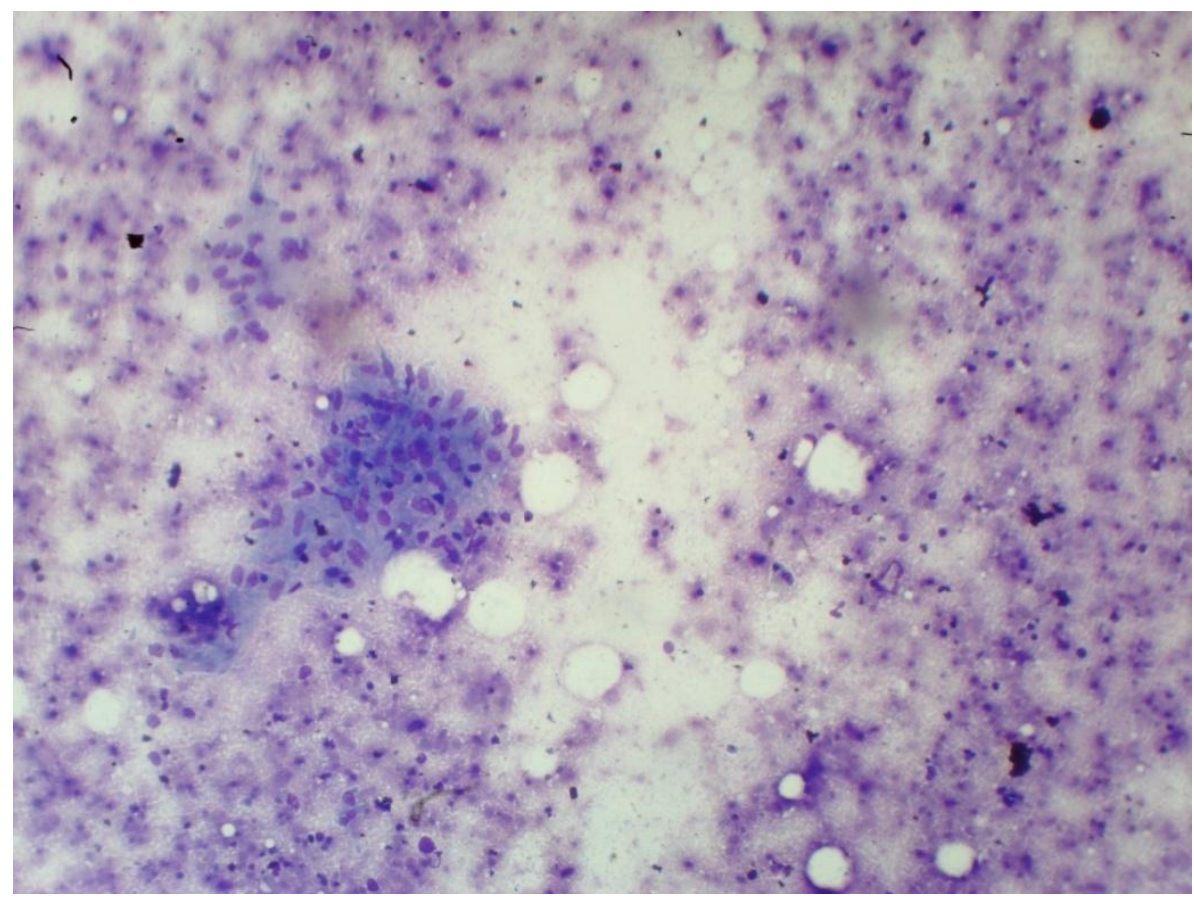

Figure showing tubercular granuloma MGG stain 40x 


\section{References}

1. Refe intro- Kumar V, Abbas AK, Fausto N, Mitchell RN (2007).Robbins Basic Pathology (8th ed.). Saunders Elsevier. pp. 516-522. ISBN 978-1-4160-2973-1.

2. Kumar A. Lymph node tuberculosis. In: Sharma SK, Mohan A. eds (2009) Tuberculosis. 2nd ed. New Delhi: Jaypee Brothers Medical Publishers, 397-409.

3. Global tuberculosis control: surveillance, planning, financing: WHO report 2008.

4. "Tuberculosis Fact sheet $\mathrm{N}^{\circ} 104 "$. World Health Organization. November 2010. Retrieved 26 July 2011.

5. Tuberculosis World Health Organization. 2002.

6. World Health Organization (2009). "Epidemiology" (PDF) Global tuberculosis control: epidemiology, strategy, financing. pp. 6-33. ISBN 978-92-4-156380-2.

7. "Improved data reveals higher global burden of tuberculosis". who.int. 22 October 2014. Retrieved 23 October 2014.

8. GBD 2013 Mortality and Causes of Death, Collaborators (17 December 2014). "Global, regional, and national age-sex specific all-cause and cause-specific mortality for 240 causes of death, 19902013: a systematic analysis for the Global Burden of Disease Study 2013". Lancet 385 (9963):

$117-$

171.doi:10.1016/S0140-6736(14)61682-

2. PMC 4340604. PMID 25530442.

9. World Health Organization (2011). "The sixteenth global report on tuberculosis" (PDF).

10. Lawn, SD; Zumla, AI (2 July 2011). "Tuberculosis". Lancet 378 (9785): 5772. doi:10.1016/S0140-6736(10)621733.PMID 21420161.

11. Dolin, [edited by] Gerald L. Mandell, John E. Bennett, Raphael (2010). Mandell, Douglas, and Bennett's principles and practice of infectious diseases ( 7 th ed.). Philadelphia, PA: Churchill Livingstone
/Elsevier. pp. Chapter 250. ISBN 978-0443-06839-3.

12. al.], edited by Peter G. Gibson; section editors, Michael Abramson ... [et (2005). Evidence-based respiratory medicine (1. publ. ed.). Oxford: Blackwell. p. 321. ISBN 978-0-7279-1605-1.

13. Jindal, editor-in-chief SK (2011). Textbook of pulmonary and critical care medicine. New Delhi: Jaypee Brothers Medical Publishers. p. 549. ISBN 978-93-5025-073-0.

14. Golden MP, Vikram HR (2005). "Extrapulmonary tuberculosis: an overview".American Family Physician 72 (9): 1761-8. PMID 16300038

15. Backer AID, Mortele KJ, Keulenaer BLD, Parizel PM (2006) Tuberculosis: epidemiology, manifestations, and the value of medical imaging in diagnosis. JBR-BTR 89: 243-250.

16. Bailey and Love's Short Practice of Surgery. 19th Edition, 1985, pages 601 and 610.

17. Ergete $\mathrm{W}$ and Bekele A. Acid fast bacilli in aspiration smears from tuberculous patients. Ethiop J Health Dev 2000; 14(1): 99-104.

18. Ahmad SS, Akhtar S, Akhtar K, Naseem S, MansoorT, Khalil S. Incidence of tuberculosis from study of fine needle aspiration cytology in lymphadenopathy and acid fast staining. Ind $\mathrm{J}$ Community Medicine 2005; 30(2):63-5.

19. RuchiKhajuria, KC Goswami, K Singh, VK Dubey. Pattern of Lymphadenopathy on Fine Needle Aspiration Cytology In Jammu. JK Science. 2006;190(3):158-60.

20. Nidhi Paliwal, Sapna Thakur, Shalini Mullick, Gupta Kumud. FNAC In Tuberculosis Lymphadenitis : Experience From A Tertiary Level Referral Centre. Indian J Tuberc. 2011;58:102-07

21. Nitin Chawla, Sanjeev Kishore, Sandip Kudesia. FNAC of Lymph Node 
Disorders. . Indian Medical Gazette. 2012:312-15.

22. Raghab Mohapatra Prasanta, Kumar Janmeja Ashok. Tuberculosis Lymphadenitis. JAPI. 2009;57:585-90.

23. RK Narang, S Pradhan, RP Chaturvedi, S Sheridan. Place of Fine Needle Aspiration Cytology In The Diagnosis of Lymphadenopathy. Ind J Tub. 1990;37: 29-31.

24. I Gomes, E Trindade, O Vidal, et al. Diagnosis of sputum smear-negative forms of pulmonary tuberculosis by transthoracic fine needle aspiration. Tubercle. . 1991;72:210-13.

25. DK Das, JN Pank, KL Chachra, et al. Tuberculosis lymphadenitis: correlation of cellular components and necrosis in lymph node aspirate with AFB positivity and bacillary count. Indian J Pathol Microbiol. 1990;33:1-10. 